\title{
Tambours et pouvoirs au Tibesti (Tchad)
}

\section{Monique Brandily}

\section{(2) OpenEdition \\ Journals}

Édition électronique

URL : http://journals.openedition.org/ethnomusicologie/2391

ISSN : 2235-7688

Éditeur

ADEM - Ateliers d'ethnomusicologie

Édition imprimée

Date de publication : 1 janvier 1990

Pagination : 151-160

ISBN : 2-8257-0423-7

ISSN : $1662-372 X$

Référence électronique

Monique Brandily, "Tambours et pouvoirs au Tibesti (Tchad) », Cahiers d'ethnomusicologie [En ligne],

3| 1990, mis en ligne le 15 octobre 2011, consulté le 19 avril 2019. URL : http://

journals.openedition.org/ethnomusicologie/2391 


\title{
TAMBOURS ET POUVOIRS AU TIBESTI (Tchad)
}

\author{
Monique Brandily
}

Les tambours du Tibesti sont de trois types nommés respectivement $k w e l l i$, nang'ara et kidi. Ils appartiennent à deux catégories bien distinctes qui se différencient par la facture, la technique de jeu, la fonction et, plus encore, par le statut de ceux qui sont, ou ne sont pas, admis à les frapper. Il en résulte, bien évidemment, des différences importantes dans le domaine de la relation avec les divers genres de pouvoirs, qu'ils soient affichés ou occultes.

Le $k$ welli et le nang'ara constituent l'une des catégories, le kidi l'autre. On précisera chaque fois ce que l'on peut entendre par «pouvoir», terme équivoque s'il en est puisque le concept qu'il recouvre varie avec les caractéristiques des institutions au sein desquelles il se concrétise. Sans entrer dans le détail, compte tenu de la place impartie, trois aspects seront retenus ici: le pouvoir sur les tambours et la façon dont il s'exprime; le signe de pouvoir exercé ou subi que constitue le jeu des tambours; enfin les pouvoirs d'ordre divers qui sont reconnus aux tambours eux-mêmes lorsqu'ils résonnent.

Quelques informations sur les grands traits de la société du Tibesti sont un préalable indispensable à la compréhension du rôle qu'y jouent les instruments en question. Le massif du Tibesti, situé à l'extrême nord du Tchad dans le Sahara oriental, est peuplé par les Teda, «nomades noirs du Sahara» ainsi que les définit Jean Chapelle (1957, rééd. 1982). Plus connus sous le nom de Toubou, ils sont islamisés mais ont conservé des institutions originales qui les distinguent des populations environnantes, en particuliers de celles qui sont de culture arabe. On n'en retiendra, bien entendu, que les traits qui sont directement en relation avec notre sujet. La société est divisée en trois strates endogames: les Teda proprement dits, au sommet de la hiérarchie; les kamaja, qui ont des ancêtres captifs, proches ou lointains, mais dont certains sont là depuis un grand nombre de générations et sont totalement intégrés culturellement; enfin les forgerons, duudé. L'appartenance à l'un ou à l'autre de ces groupes est déterminante pour accéder au jeu des différents tambours dont on donnera une description succinte. 


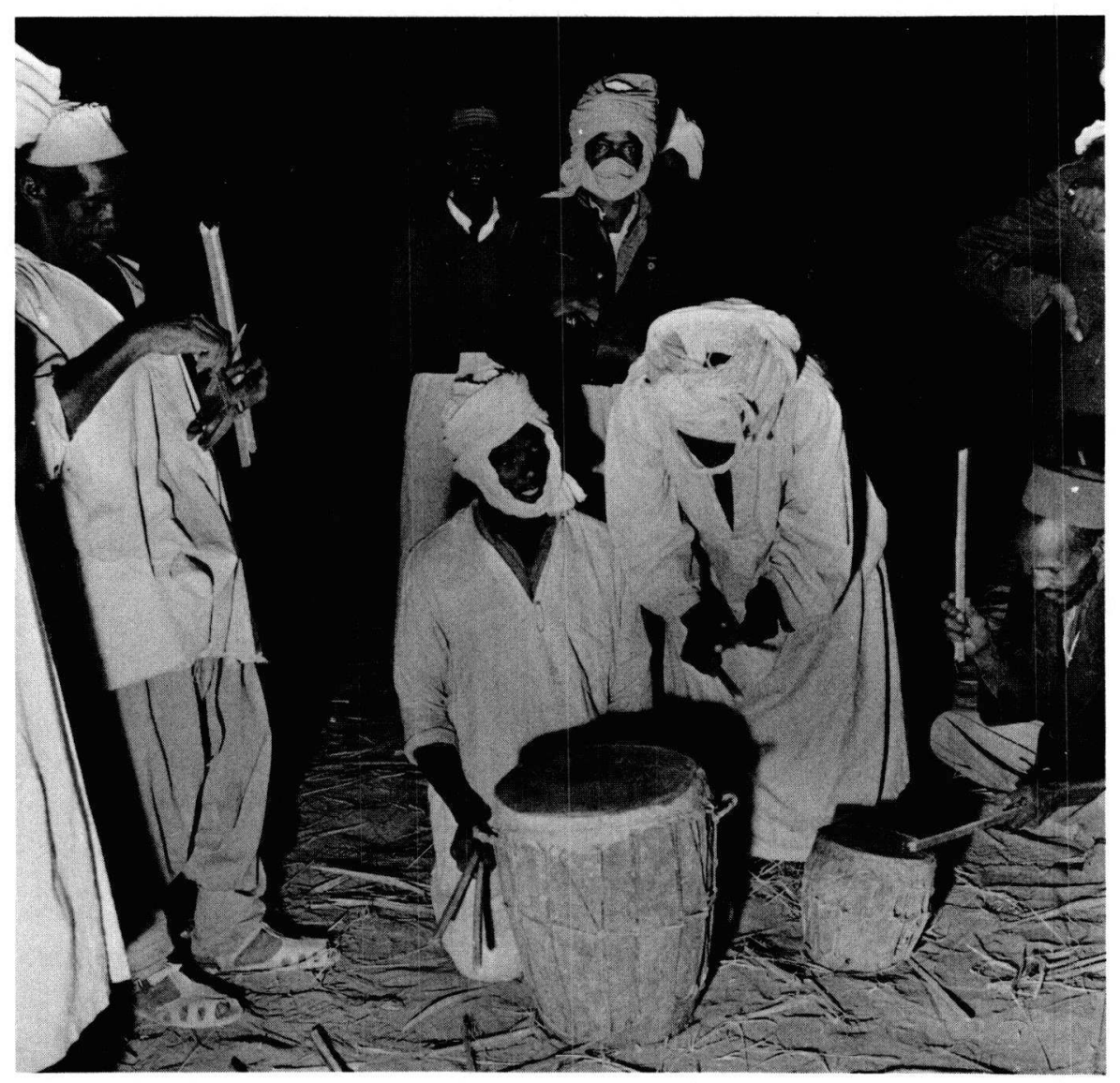

Fig. 1: Préparatifs et taille des baguettes avant le jeu des tambours nang'ara et kwelli. Photo: M.-Y Brandily.

\section{Le kwelli}

C'est un petit tambour à deux membranes dont la hauteur moyenne est d'environ trente centimètres. La caisse de résonance est creusée dans une bille de bois dont on a enlevé l'écorce et dont l'une des extrémités est taillée de telle façon que l'ensemble prenne une forme ovoïde. L'un des orifices est donc sensiblement plus petit que l'autre. Les membranes qui recouvrent ces orifices, en peau de chameau séchée, sont fixées par laçage. En position de jeu, le tambour est accroché ou posé verticalement, la membrane la plus petite en bas; seule la plus grande, placée au dessus, est frappée au moyen de baguettes droites utilisées fraîchement coupées afin qu'elles conservent une certaine souplesse. Le corps de résonance, dans lequel on perce un trou au fer rougi au feu, est le plus souvent en bois d'acacia mâle. 


\section{Le nang'ara}

Sa facture générale est identique à celle du kwelli, dont il ne diffère que par ses dimensions qui sont environ le double. Pour en jouer, on le suspend également à un piquet fourchu ou on le place sur une selle de chameau ou encore on le pose simplement sur le sol en ayant soin de le placer en porte-à-faux sur un caillou pour que la membrane inférieure puisse vibrer par sympathie en cours de jeu.

\section{Le kidi}

Il est très différent des précédents bien qu'il s'agisse également d'un tambour à deux peaux. Il se joue porté en bandoulière et sa caisse est creusée dans un bois beaucoup plus léger que l'acacia, généralement du bois d'euphorbe. La forme de cette caisse, plus allongée que celles du nang'ara et du kwelli, est cylindrique sur les deux tiers de sa longueur et tronconique sur le dernier tiers. Là encore, on dispose donc de deux membranes de dimensions inégales. Elles sont en peau de chèvre ou de gazelle, sensiblement moins épaisse que la peau de chameau et elles sont toutes deux frappées à mains nues. On y applique des pastilles de goudron plus ou moins épaisses pour obtenir la sonorité souhaitée.

Le kidi est joué exclusivement par les forgerons dont il accompagne le chant; il constitue l'une des marques les plus visibles de leur condition. Aucun homme n'appartenant pas à leur caste n'aurait l'idée de chanter au village, pas plus que de jouer du kidi. Ces deux activités étant le signe d'une situation de dépendance par rapport au groupe dominant dans son ensemble et, à plus forte raison, par rapport à ceux qui y sont investis d'une autorité à un degré quelconque.

En contrepartie, il faut remarquer que la maîtrise de la fabrication du kidi, de son jeu et de la musique qui lui est liée confère aux forgerons des pouvoirs d'un autre ordre. L'un d'eux s'exerce sur le psychisme de ses auditeurs qui sont valorisés, confortés et, en quelque sorte, fortifiés par les louanges adressées publiquement à eux-mêmes ou à leur famille. Etre le sujet de louanges proférées en chantant et solennisées par le frappement d'un tambour est aussi, indirectement, l'affirmation de la situation prééminente du louangé, et cela en présence des femmes, attentives aux paroles du musicien qu'elles enserrent dans leur cercle dansant. Un autre pouvoir ressortit au domaine social stricto sensu. En effet, si, contrairement à ce que l'on observe dans certaines ethnies des régions voisines, le forgeron du Tibesti ne se permet pas la moquerie envers ceux qui manifestent trop de ladrerie à son égard, en revanche, le fait de passer sous silence ou de chanter très brièvement sur telle ou telle personne revient à faire passer le même message clairement, encore qu'il reste du domaine du non-dit.

Le statut et le rôle du nang'ara et du kwelli apparaissent infiniment plus complexes, ce qui s'explique par le fait qu'ils sont beaucoup plus étroitement liés au fonctionnement de la société traditionnelle. 
Ces deux tambours, à l'inverse du kidi, sont volontiers frappés par les hommes appartenant à la caste placée au niveau supérieur de la hiérarchie sociale. Leur jeu est soumis à une réglementation strictement observée. Il est réservé aux hommes socialement adultes tandis qu'il est interdit aussi bien aux femmes qu'aux garçons non encore circoncis (cette opération marquant l'entrée dans l'âge socialement considéré comme adulte, quel que soit l'âge biologique).

Les jeunes garçons non circoncis s'exercent à maîtriser les rythmes qu'ils exécuteront plus tard sur les tambours en frappant sur une grosse branche dont ils ont détaché et soulevé une partie de l'écorce. L'instrument ainsi réalisé ne doit pas être identifié à une cithare sur bâton, comme sa facture pourrait y inciter, car sa fonction et son utilisation le désignent sans ambiguïté comme le simple substitut du grand tambour et du kwelli.

Du fait même de leur insertion institutionnelle, l'usage de ces tambours s'est modifié quelque peu en raison des changements intervenus au cours de l'histoire récente, soit celle qui coïncide avec la période coloniale, qui a apporté un certain assouplissement de la stratification sociale et de ses marques sans jamais la supprimer. Cette période, rappelons-le, a été particulièrement brève dans cette partie du Tchad. Le Tibesti, toujours resté sous administration militaire, n'a connu le poids de garnisons permanentes (peu nombreuses et composées d'effectifs restreints) qu'à partir de 1932 hormis un bref épisode interrompu par la Première Guerre mondiale. Les anciennes manières de vivre se sont donc maintenues jusqu'à une époque proche de la nôtre, et le souvenir en est resté très présent. C'est le cas, notamment, pour les expéditions guerrières destinées à se procurer du bétail et des captifs, et dans la conduite desquelles le tambour kwelli jouait un rôle important et double. En effet, le chef de l'expédition le fixait à la selle de son chameau et le frappait pour indiquer à ses compagnons, par exemple, le moment de se mettre en route ou de se replier; le kwelli avait donc à la fois une fonction de marqueur, puisque seul le chef en disposait, et d'instrument de communication. Il assumait ce dernier rôle dans d'autres circonstances également; dans les villages, si quelque information importante devait être transmise à l'ensemble des habitants, on frappait le kwelli pour les rassembler. Mise à part son utilisation en déplacement, il peut être remplacé par le nang'ara. Ce dernier, cependant, a une fonction qui lui est propre et qui est très directement liée au pouvoir traditionnel, au sens banal du terme.

Lors de l'investiture d'un nouveau derdé (ainsi nomme-t-on le chef traditionnel de l'ensemble du Tibesti), l'un des premiers actes accomplis quand commence la préparation de la cérémonie est la fabrication d'un tambour nang'ara neuf. Le rôle de ce dernier est essentiel dans la mesure où, après la déclaration d'acceptation du pouvoir, l'impétrant n'en est réputé effectivement investi qu'à l'instant où retentit le troisième coup frappé sur le nouveau tambour par l'intéressé lui-même. Cette frappe se fait au moyen d'une seule baguette tenue dans la main droite. Elle est a-musicale.

A l'inverse, quand le soir est venu, commence la fête autour du même tambour qui, cette fois, est frappé musicalement par deux jeunes hommes 


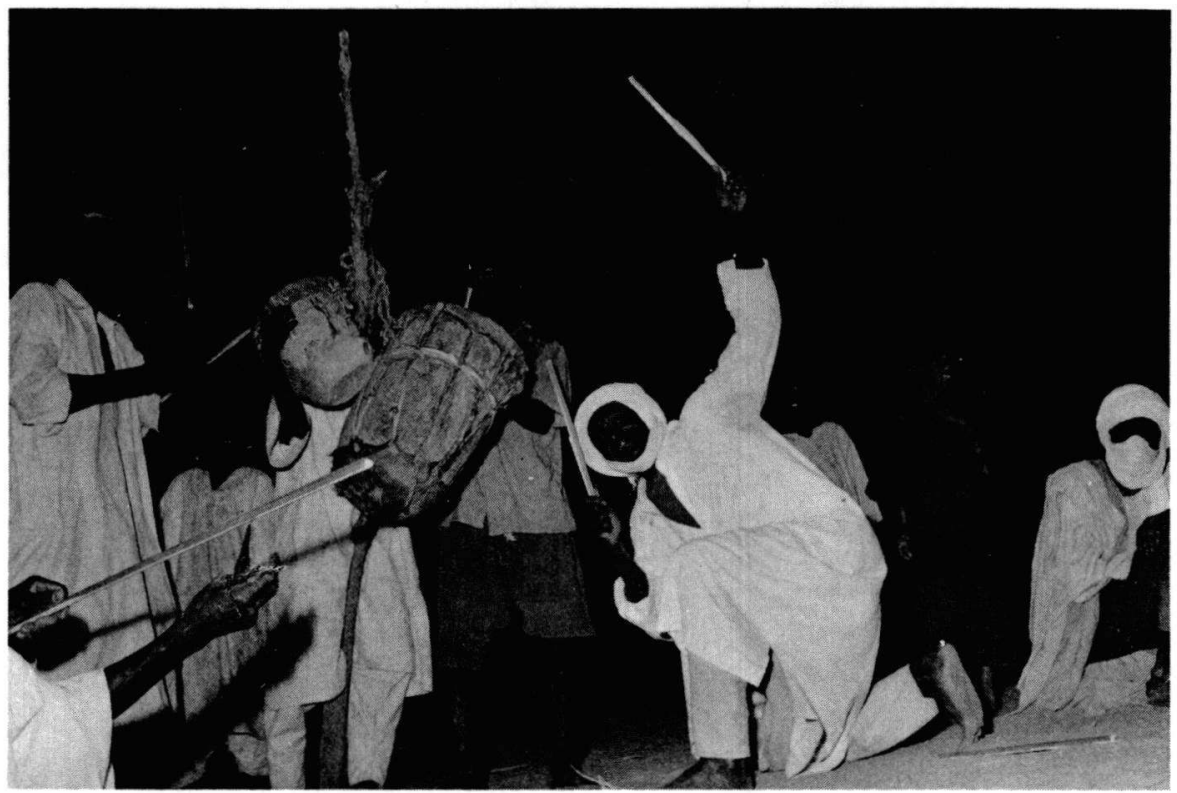

Fig. 2: Quand on frappe le rythme digidèl sur les tambours nang'ara et kwelli couplés, l'un des trois tambourinaires se livre à une gesticulation inhabituelle chez les Teda, hormis cette circonstance. Photo: M.-Y Brandily.

ensemble dont chacun utilise deux baguettes tandis que les jeunes filles, sur le rythme ainsi réalisé, chantent les louanges des hommes de leurs familles respectives.

Certaines batteries, désignées par une onomatopée: digidèl nécessitent la participation de trois tambourinaires. Dans ce cas, les deux tambours nang'ara et kwelli sont placés côte à côte. Deux des tambourinaires frappent le nang'ara tandis que le troisième frappe le kwelli. Cette batterie, jugée plus ancienne, était celle qui célébrait les retours d'expédition avant la «paix coloniale».

Quant à la frappe à une seule baguette, destinée à marquer irréversiblement le moment précis auquel une parole solennelle prend effet, elle intervient encore quand il y a eu meurtre. Si la famille de la victime, à la suite de longues tractations, a accepté de renoncer à exercer son droit ou, plus exactement, son devoir de vengeance à l'encontre du meurtrier lui-même ou, à défaut, de l'un de ses proches, on procède à la remise de la compensation: gueregué, le prix du sang. Il s'agit généralement de chameaux pour la fourniture desquels toute la famille s'est cotisée. Lorsque l'opération est terminée, en présence du derdé, on frappe le tambour pour marquer l'instant à partir duquel l'obligation et le droit de vengeance sont éteints.

Ces exemples illustrent conjointement deux des aspects que nous nous proposions de souligner. D'une part le tambour comme signe du pouvoir ou, pour mieux dire, de l'autorité du derdé (dont le rôle est celui d'un arbitre plutôt que 
d'un chef autocratique) et, d'autre part, le pouvoir du tambour lui-même qui donne à la parole force de loi lorsqu'il a résonné. Cette dernière précision n'est pas superflue dans la mesure où, ailleurs, certains instruments de musique sont porteurs de signification en eux-mêmes, indépendamment du son qu'ils sont aptes à produire. En la matière, il semble que le degré extrême soit atteint par certains des tambours royaux du Rwanda dits «tambours dynastiques» ingabé. Ils étaient la représentation du pouvoir royal mais n'étaient jamais joués (Gansemans 1988: 185).

Au Tibesti, comme on l'a vu, le pouvoir des tambours s'exprime de façon sonore mais selon une modalité qui exclut toute confusion entre ces sons efficients et ceux qui appartiennent au domaine musical en coordination avec le chant féminin.

Encore convient-il de souligner que ces deux rôles ne sont pas aussi différents l'un de l'autre qu'une vue ethnocentriste occidentale pourrait le donner à penser. En effet, rappelons que, jusqu'à une époque récente, les principales occasions de fête, donc de réunions de musique et de danse autour des tambours, étaient les retours d'expéditions, sources d'enrichissement majeures, et que le chant des femmes y célébrait la valeur des hommes. Leurs actes de bravoure étaient donc proclamés publiquement et la frappe du tambour, là aussi, donnait à ces paroles une solennité et un statut qui les intégraient dans la tradition orale, c'est-à-dire dans le patrimoine social. Même depuis que ce genre d'expéditions n'est plus pratiqué, la fonction correspondante du tambour n'a pas entièrement disparu puisque le thème le plus habituel des chants féminins reste la louange des hommes et l'énumération de leurs qualités et de leurs richesses, ces richesses qui, ne l'oublions pas, étaient avant tout le signe immédiatement perceptible des qualités viriles nécessaires pour les acquérir à la pointe de la sagaie.

Il ressort de tout cela que la frappe musicale, tout comme la frappe a-musicale de l'instrument, est en relation de coordination avec la parole chantée dans un cas, parlée (ou criée) dans l'autre et témoigne du pouvoir qu'a le tambour de donner aux paroles qu'il soutient une portée sociale qui opère immédiatement la distinction entre elles et la parole du domaine privé ou de l'intimité.

Il est intéressant d'observer qu'il existe également une opposition entre les émissions vocales utilisées suivant qu'il s'agit de l'une ou l'autre catégorie de paroles. Qu'elles soient chantées (par les femmes) ou parlées-criées (par les hommes), les paroles «tambourisées» sont émises à voix forte alors que la bienséance, dans cette société, exige que l'on parle à voix plutôt confidentielle ou même que l'on s'abstienne de parler, notamment lorsqu'on est dans un lieu public dans les circonstances de la vie quotidienne.

S'il semble admis que le pouvoir du tambour est apparenté au pouvoir de la parole et que les deux, en étroite association, doivent converger pour atteindre une pleine efficacité, à l'inverse, l'idée que l'on puisse réaliser un langage intelligible avec les tambours semble farfelue dans la mesure où ce sont les instruments à cordes, ceux qui sont capables de dessiner des mélodies, qui sont jugés aptes à exprimer des messages équivalents à ceux de la parole chantée. 
Il reste à donner quelques indications sur ceux qui détiennent un pouvoir sur les tambours et la façon dont cela s'exprime. Dans une société comme celle du Tibesti où l'individualisme est très valorisé, la notion de pouvoir autoritaire semble tout à fait irrecevable à l'ensemble de la population, et la disposition des tambours n'échappe pas à cette manière de voir.

Pour apprécier le pouvoir exercé sur les instruments, on peut retenir trois critères: la garde, l'appropriation et l'usage.

Pour aller d'abord au plus simple, disons que la garde n'est pas réglementée. La charge peut en être confiée à quiconque accepte cette responsabilité, y compris à quelqu'un qui ne serait pas admis à frapper ces tambours, notamment une femme. J'en ai observé des exemples concrets. Comme on le sait, cette possibilité est loin d'être universelle.

En ce qui concerne l'appropriation, tout ce qui va être dit du nang'ara s'applique au kwelli, hormis le cas particulier du nang'ara du derdé sur lequel on va revenir. En effet, à la différence de ce qui a souvent été décrit ailleurs, la possession du nang'ara n'est pas l'apanage exclusif du derdé. Presque chaque village a son tambour; je dis «presque» car il arrive que l'un d'eux soit abîmé et que l'on n'ait pas le temps de le remettre en état, auquel cas rien n'empêche d'emprunter celui d'un village voisin. C'est la population du village dans son ensemble qui en est propriétaire. Quand on décide d'en construire un neuf, la décision et la recherche du bois adéquat sont collectives; l'exécution en est confiée à un homme jugé habile et le fait que ce soit un descendant de captif n'y fait pas obstacle. Il en sera de même lorsqu'il deviendra nécessaire d'en changer les membranes.

L'appropriation du nang'ara et du kwelli est donc de type collectif, la collectivité concernée reposant non sur la consanguinité ou le clan, mais sur l'implantation territoriale. Cela ne laisse pas de surprendre de la part d'une population classée parmi les nomades. Il faut dire que les règles très strictes prohibant les mariages consanguins jusqu'au quatrième degré obligent le plus souvent les jeunes hommes à prendre épouse loin de leur entourage de naissance. C'est la cause d'un brassage de population en fonction duquel la relation de voisinage, qui crée des intérêts communs dans la vie de tous les jours, prend une importance pratique considérable.

Ceci explique le statut singulier du tambour du derdé: ce dernier étant le représentant du peuple de la totalité du Tibesti, son tambour se trouve également rattaché à l'ensemble de ce territoire à travers lequel on est souvent amené à le transporter et où il est partout efficace.

On perçoit immédiatement la différence majeure entre cet aspect institutionnel et les tambours et timbales régalia que l'on trouve un peu partout dans le monde islamisé (Schaeffner 1952) et, singulièrement, chez des populations proches de celles du Tibesti: Kanem, Bornou, Ouadaï où, pour les sultans Zaghawa «s'en laisser déposséder équivalait à perdre son titre » (Tubiana 1964: 36). On a déjà mentionné plus haut les tambours dynastiques des anciens souverains du Rwanda qui, s'ils étaient pris par l'ennemi, signifiaient la victoire de celui-ci. Il en allait de même, sauf erreur de ma part, pour les souverains du 
Burundi. L'Islam a, sans doute, privilégié certains types instrumentaux, mais le rôle régalien attribué à des instruments de musique dépasse largement les limites de l'expansion islamique.

Enfin, si l'on considère le pouvoir de frapper le nang'ara et le kwelli, il faut distinguer selon qu'il s'agit d'en faire un usage musical ou de mettre en jeu la fonction exclusivement ratificatrice dont le nang'ara en particulier est investi pour entériner et solenniser une parole efficace.

Pour l'usage musical, hormis l'obligation déjà signalée d'être un homme socialement adulte (i.e. déjà circoncis), l'accès au jeu des tambours est réputé libre. Tous les jeunes hommes sont valorisés s'ils sont bons tambourinaires. Bien souvent, on voit des jeunes appartenant à la catégorie des kamaja qui y excellent et sont appréciés dans ce rôle, animant la réunion en rendant plus vibrant le chant des jeunes filles, toutes catégories confondues chez elles également.

Il faut cependant ajouter que, si le principe du libre accès aux tambours pour tous les hommes adultes m'a été maintes fois confirmé verbalement, dans la pratique je n'ai jamais vu un forgeron frapper un nang'ara ou un kwelli. Rappelons que le jeu de ces deux instruments est une activité qui ne saurait en aucun cas comporter un aspect vénal. Cela constitue une différence majeure avec le jeu du kidi, réservé aux forgerons, largement rémunéré. De ce point de vue, il est évidemment plus intéressant pour eux de consacrer leurs soirées à cette activité lucrative. On peut cependant se demander s'il ne s'y ajoute pas quelque inhibition d'un autre ordre, explicable historiquement mais qui reste inexprimée.

Au quotidien, dans la région de Bardaï au centre du massif, quand le Tibesti était encore administré par les Français et encore après leur départ, les réunions de danse autour des forgerons se tenaient dans le village de Youyour, proche des bâtiments administratifs, et les membres de la garnison aussi bien que les visiteurs occasionnels y assistaient souvent.

Dans le même temps les Teda organisaient des réunions de musique et de danse $a b i$ entre eux, dans un village voisin (souvent, à cette époque, à Armachibé distant de deux à trois kilomètres du centre de Bardaï). Ils frappaient le nang'ara, seul ou jumelé avec le kwelli, accompagnant le chant et la danse des jeunes filles.

Une telle répartition géographique illustrait et accentuait, s'il en était besoin, la différence de statut qui sépare les deux catégories de tambours et cela d'autant plus que, dans un cas, ce sont les jeunes filles qui chantent tandis que dans l'autre, c'est le joueur de tambour kidi lui-même. Or, ce comportement serait parfaitement honteux pour tout homme autre que le forgeron.

$\mathrm{Si}$, comme on vient de le voir, le clivage est net entre le kidi d'une part et, de l'autre, le nang'ara et le kwelli dans leurs usages respectifs d'ordre musical, il va sans dire que, lorsqu'on considère la fonction, propre au nang'ara du derdé, de confirmer en la rendant irréversible une déclaration vitale pour la collectivité, l'écart est tel que l'on s'attendrait à voir utiliser un autre instrument. Pour les Teda, cependant, il semble que la façon de le faire sonner et les 


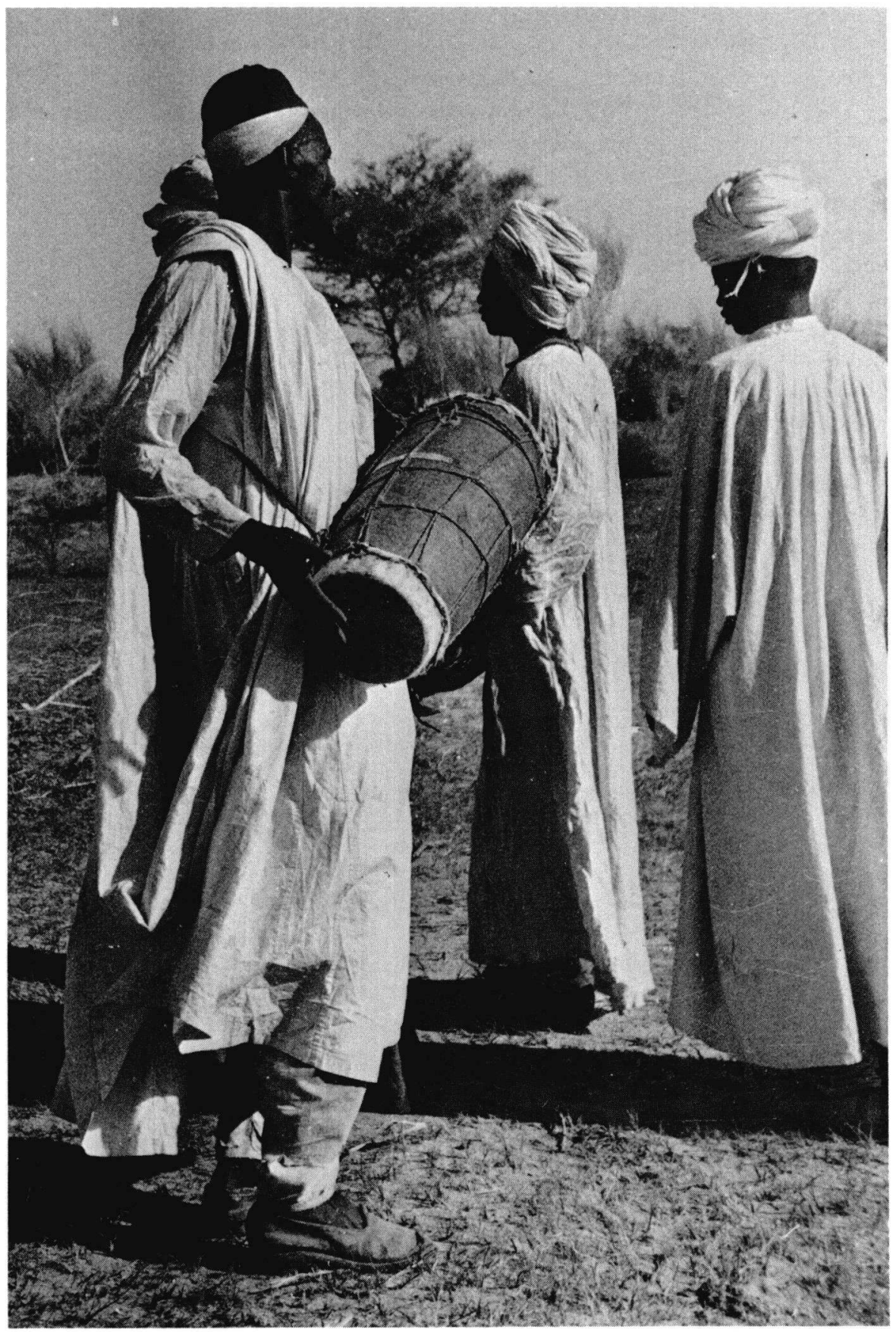

Fig. 3: Le tambour de forgeron kidi en cours de jeu. On voit la pastille de goudron appliquée sur la partie centrale de la peau. Photo: M.-Y Brandily. 
circonstances d'utilisation autant que le statut de la personne habilitée à le frapper soient des éléments jugés largement suffisants pour différencier les deux fonctions sans aucun risque de confusion.

En guise de conclusion aux quelques remarques qui précèdent et qui sont loin d'épuiser le sujet, on ajoutera simplement deux observations.

La première est que l'on y trouve la confirmation qu'un rôle social tout à fait important peut se voir assumé par des instruments qui, au premier regard, ne semblent pas faire l'objet d'une vénération particulière et sont d'une facture éventuellement rustique.

La seconde est pour souligner la pluralité des formes que les sociétés humaines ont élaborées pour exprimer la charge symbolique confiée par elles à divers objets sonores, épuisant, semble-t-il, tous les cas de figure: l'objet indépendamment du son qu'il peut produire, la forme, le matériau, les ornements, les couleurs ou encore le son seul d'un objet qui reste caché à la vue, comme c'est le cas de nombreuses sociétés initiatiques.

Le trait commun pertinent étant la faculté de bruire qui en fait un objet «à part» puisqu'il possède l'un des attributs du vivant qui est de disposer d'une voix.

\title{
Bibliographie
}

\author{
BRANDILY Monique \\ 1974 Instruments de musique et musiciens instrumentistes chez les Teda du Tibesti. Tervuren: \\ Musée royal de l'Afrique centrale (MRAC). \\ 1981 «Tibesti, l'intronisation du Derdé» Balafon 51: 12-21. \\ 1988 «Les inégalités dans la société du Tibesti»In: Catherine Barouin, éd. Gens du roc et du \\ sable-les Toubous. Paris: Presses du CNRS. \\ CHAPELLE Jean \\ 1982 Nomades noirs du Sahara. Paris: L'Harmattan. [Rééd. de l'ouvrage de 1957, Paris: Plon]. \\ GANSEMANS Jos \\ 1988 Les instruments de musique du Rwanda. Tervuren: MRAC. \\ SCHAEFFNER André \\ 1952 «Timbales et longues trompettes» Bulletin de l'IFAN 14 (4) : 466-89. \\ TUBIANA M.-J. \\ 1964 Survivances préislamiques en pays Zaghawa. Paris: Institut d'ethnologie.
}

